\title{
Achieving Service Differentiation and High Utilization in IEEE 802.11*
}

\author{
Vasilios A. Siris ${ }^{\star \star}$ and Matina Kavouridou \\ Institute of Computer Science (ICS) \\ Foundation for Research and Technology - Hellas (FORTH) \\ P.O. Box 1385, GR 71110 Heraklion, Crete, Greece \\ \{vsiris,mkavouri\}@ics.forth.gr
}

\begin{abstract}
Service differentiation in wireless LANs is a growing demand, due to their increasing use for applications with different requirements and the scarcity of wireless channel resources. The contribution of this paper is twofold: First, we investigate how various parameters of the IEEE 802.11 MAC protocol affect service differentiation, in terms of both throughput and delay. Second, we propose a new approach for providing service differentiation, while achieving high wireless network utilization. Simulation investigations demonstrate that our approach can effectively adapt to varying network conditions. The approach can be implemented at the access point of a wireless LAN, while the wireless stations need only to support the emerging IEEE 802.11e standard.
\end{abstract}

Keywords: weighted fairness, throughput monitoring, wireless LANs

\section{Introduction}

The wireless LAN (WLAN) area is a field of wide development over the last few years. The two main WLAN standards are the ETSI High Performance European Radio (HIPERLAN) and the IEEE 802.11 WLAN, with the latter dominating the market. In IEEE 802.11, the primary media access mechanism is the CSMA/CA (Carrier Sense Multiple Access with Collision Avoidance) algorithm, which currently lacks support for service differentiation.

The capacity of wireless networks is much smaller than that of fixed networks. Moreover, unlike fixed networks, there is a limited ability for increasing the wireless channel capacity. At the same time, there is an increasing use of wireless networks for multimedia and delay sensitive applications, and an increasing deployment of wireless LAN hotspots. For all these reasons, it is becoming increasingly important to support service differentiation in wireless LANs.

The contribution of this paper is twofold. First, we investigate mechanisms for supporting service differentiation in 802.11 networks in terms of both throughput

* Part of this work was supported by British Telecommunications (BT), UK.

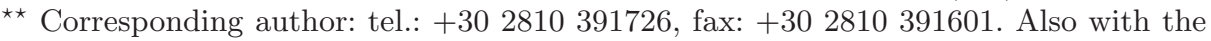
Department of Computer Science, University of Crete. 
and delay. Moreover, we investigate the weighted fairness of the various schemes, by presenting the results as the ratio of throughput achieved for the corresponding ratio of values of the control parameter. Second, we propose and investigate a new approach for supporting service differentiation, while at the same time achieving high wireless network utilization. The approach involves dynamically adjusting parameters of the 802.11 MAC protocol, to track varying conditions of the network load. The approach can be implemented solely at the access point of an 802.11 network, without requiring changes to the wireless stations; the latter need only to support the emerging IEEE 802.11e standard.

This rest of the paper is organized as follows. In Section 1.1 we briefly describe the CSMA/CA protocol, including the extensions being developed for IEEE 802.11e. In Section 1.2 we review related work on supporting service differentiation in wireless LANs. In Section 2 we investigate how various parameters of the 802.11 MAC affect service differentiation, in terms of both throughput and delay. In Section 3 we present and investigate a new approach for achieving service differentiation while attaining high network utilization. Finally, in Section 4 we present some concluding remarks and identify areas for further investigation.

\subsection{IEEE 802.11}

The IEEE 802.11 MAC supports two access modes: Distributed Coordination Function (DCF) and Point Coordination Function (PCF). In the first, which is the primary mode and best suited for traffic without strict delay requirements, wireless stations contend for the wireless channel at every frame transmission. The basic scheme for DCF is Carrier Sense Multiple Access with Collision Avoidance (CSMA/CA). A collision can be caused by two or more stations trying to transmit at the same time. After each frame transmission, the sender waits for an acknowledgment (ACK) from the receiver. If no ACK is received, a collision must have occurred and the frame is retransmitted.

Frames can have different priorities by varying the time interval, called interframe spacing (IFS), the channel must be sensed idle prior to their transmission. In DCF, two IFSs are defined: Short IFS (SIFS) and DCF IFS (DIFS), where SIFS is smaller than DIFS. Hence, an ACK which uses SIFS has a higher transmission success probability than a new data frame, which uses DIFS.

The objective of the collision avoidance part of CSMA/CA is to avoid simultaneous frame transmissions right after the channel is sensed idle. This can be achieved if, prior to transmitting a frame, a station waits for the channel to be idle for some random backoff time. This backoff time is selected randomly from the interval $[0, C W-1] \cdot$ slot_time, where $C W$ is the contention window, whose initial value is $C W m i n$. After each unsuccessful transmission, the contention window is doubled up to some maximum value $C W \max$.

An important activity of IEEE is its work towards 802.11 e, which aims to improve the media access mechanism and to add support for service differentiation [1]. The extensions include the Enhanced DCF (EDCF) algorithm. Although all the details have not yet been finalized, EDCF supports different classes, with different values for $C W \min , C W \max , D I F S$ (now called Arbitration IFS - AIFS), 
and the persistence factor $P F$, which determines the increase of the contention window after a collision. Smaller values of $C W \min , C W \max , A I F S$, or PF correspond to a higher priority.

\subsection{Related Work}

Next we review some representative related work, without attempting to perform an exhaustive survey of the area. The work in [2] investigates different backoff increase rates and DIFS intervals for providing service differentiation. Simulation experiments show that such schemes work well for UDP traffic, but not so well for TCP traffic. The work of [3] investigates how the above mechanisms, in addition to $C W$ min differentiation, can be adjusted to support per flow differentiation, rather than per station differentiation. In both works, differentiation is in terms of throughput, and the wireless channel utilization is not considered.

Another direction involves the development of new distributed access algorithms for supporting service differentiation 4 5|6 7/8. The algorithm proposed in [5] tries to achieve fair bandwidth distribution by setting the frame size and the initial value of the backoff interval based on weights. In the work of [6] each wireless station adjusts its contention window based on a fairness index, which measures the degree of fairness of the station's throughput, relative to some reference value. In earlier work, the MACAW protocol [4] adjusts the backoff timer using a multiplicative increase/additive decrease algorithm.

The work in [7] deals with fairness and wireless channel utilization, and proposes a scheme for setting the backoff interval for different traffic classes, based on their weights and the estimated number of contending stations. The work in [8] introduces virtual MAC and virtual source algorithms that passively monitor the radio channel to determine if the channel can support new service requests in terms of delay and loss. The schemes described in the last two paragraphs all require changes to the MAC layer running at the wireless stations.

\section{Service Differentiation Mechanisms}

In this section we investigate how parameters of IEEE 802.11, and 802.11e in particular, affect service differentiation in terms of both average throughput and delay. The parameters we consider are 1) the maximum frame size, 2) DIFS (interval the channel must be sensed idle prior to frame transmission), and 3) $C W$ min (minimum value of the contention window). Other parameters that can affect service differentiation include $C W \max$ (the maximum value for the contention window) and the persistence factor $P F$. These parameters will affect service differentiation in the presence of collisions, which should be avoided since they decrease the channel utilization. Hence, we do not discuss them further.

Our experiments were performed using the ns-2 simulator [9]. For the experiments investigating DIFS and CWmin differentiation, we used the EDCF modules developed by Atheros Communications. The procedure for dynamically adjusting the $C W m i n$ values was implemented on top of these modules. For all 


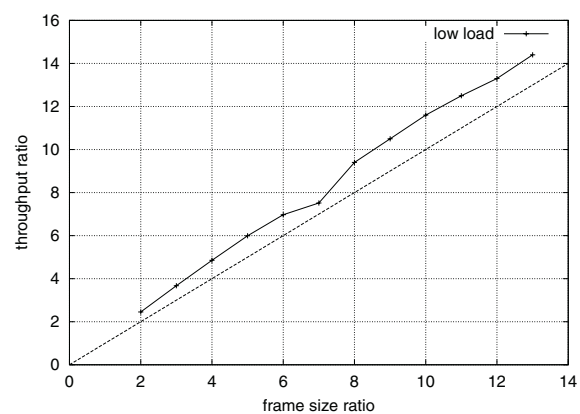

(a) Low load (=1.5 Mbps)

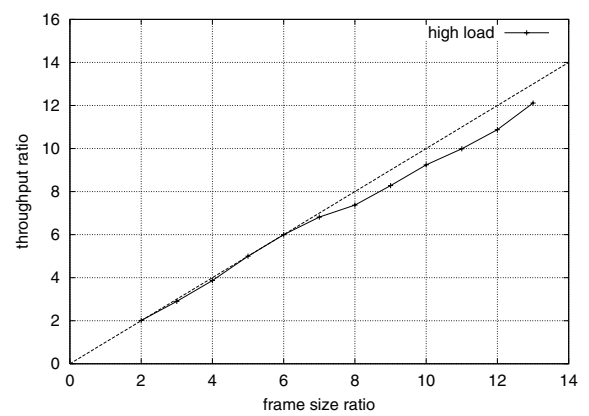

(b) High load (=2 Mbps)

Fig. 1. Service differentiation with varying maximum frame size.

experiments the channel capacity was $11 \mathrm{Mbps}$, expect for the first concerning the maximum frame size where it was $2 \mathrm{Mbps}$.

\subsection{Differentiation Based on Maximum Frame Size}

We first consider varying the maximum frame size that a station is allowed to transmit. The traffic used was constant bit rate (CBR) sources over UDP connections. The wireless LAN considered had capacity $2 \mathrm{Mbps}$, and carried 4 sources with an aggregate traffic of $1.5 \mathrm{Mbps}$. Fig. 11(a) shows that the ratio of throughput is approximately proportional to the ratio of frame sizes; each point in the graph corresponds to an experiment where the frame payload size for 2 of the sources was 300 bytes, and for the other 2, a multiple of this value. Indeed, it appears that the source with a larger frame size receives proportionally higher throughput compared to a source with a smaller frame size. This is not the case when the load is increased, as shown in Fig. 1(b), which was for 10 sources with an aggregate rate of $2 \mathrm{Mbps}$; this figure shows that sources with large frame sizes receive somewhat less capacity than that suggested by the ratio of frame sizes.

Note that varying the frame size cannot achieve delay differentiation. To achieve such differentiation, we need to use one of the other two parameters, DIFS or CWmin, which we investigate next.

\subsection{Differentiation Based on DIFS}

Next we investigate the service differentiation that is achieved with different values of DIFS, or AIFS as the inter-frame spacing interval is called in 802.11e. Our experiments involved both UDP and TCP traffic. The default value of DIFS is $34 \mu \mathrm{s}$; this value corresponds to the class with highest priority. The lower priority classes have a DIFS value larger than the default by some number of time slots, with each slot time equal to $9 \mu \mathrm{s}$.

Fig. 2(a) shows the differentiation in terms of throughput, for 5 and $10 \mathrm{CBR}$ sources over UDP connections with aggregate rate $20 \mathrm{Mbps}$ and $40 \mathrm{Mbps}$, respec- 


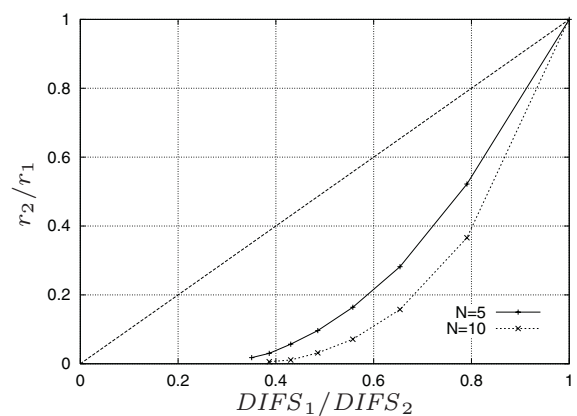

(a) CBR-over-UDP

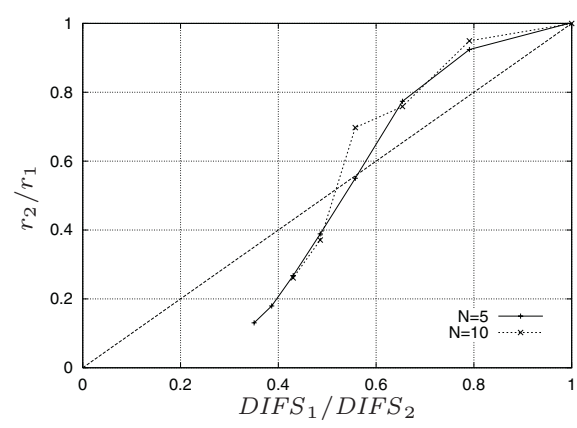

(b) TCP

Fig. 2. Throughput ratio $r_{2} / r_{1}$ as a function DIFS ratio $D I F S_{1} / D I F S_{2}$, for UDP and TCP traffic, and $N=5$ and $N=10$ sources.

tively, and payload size 500 bytes. Observe that the dependence of the throughput is far from being inversely proportional to the value of DIFS. Fig.2(b) shows that throughput differentiation in the case of TCP traffic is quite different than that for UDP traffic; indeed, for small values of DIFS, the effect of increasing DIFS is small. Also observe in Fig. 2(a) that for UDP traffic the number of sources affects the dependence of the throughput ratio on the DIFS ratio.

Fig. 3(a) shows how the average delay for voice traffic depends on the number of slots added to the default value of DIFS. The traffic consisted of 2 UDP connections carrying voice traffic, and 4 CBR sources with an aggregate bandwidth of 30 Mbps. The modelled voice traffic was assumed to be G.729 encoded: a 60 byte packet is generated every $20 \mathrm{~ms}$ during the talk spurt period, and the length of talk spurt periods is exponentially distributed with mean $352 \mathrm{~ms}$, whereas the length of silence (inactive) periods is exponentially distributed with mean 650 ms. Fig. 3(a) shows that, as expected, the average delay increases with the number of slots; indeed, the rate of increase is larger for a larger number of slots added to DIFS. Also shown in the figure is the $90 \%$ confidence interval, estimated from 10 independent runs of the experiment.

Fig. 3(b) shows the average delay of TCP traffic, in the case of 2 TCP connections, and 3 CBR sources with total bandwidth $30 \mathrm{Mbps}$. Comparison with Fig. 3a) shows that the addition of up to 6 slots results in an average delay that is the same as in voice-over-UDP traffic. The addition of more than 6 slot times results in a higher delay for voice-over-UDP traffic. Hence, the average delay appears to be less affected by DIFS for TCP traffic, compared to UDP traffic.

\subsection{Differentiation Based on $C W m i n$}

Next we investigate the differentiation achieved by varying the minimum con-

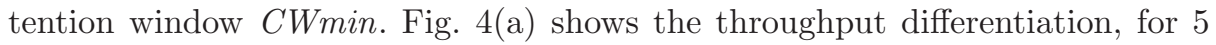
and $10 \mathrm{CBR}$ sources with aggregate rate $20 \mathrm{Mbps}$ and $40 \mathrm{Mbps}$, respectively, and payload size 500 bytes. Observe that the ratio of throughput is approximately 


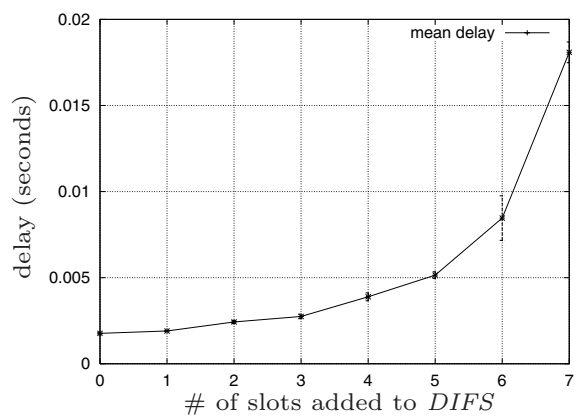

(a) Voice-over-UDP

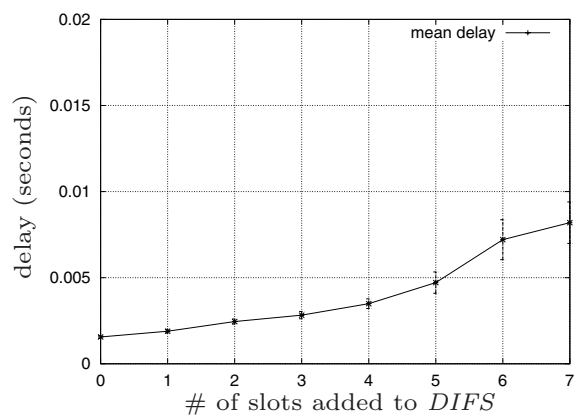

(b) TCP

Fig. 3. Delay differentiation for different DIFS values.

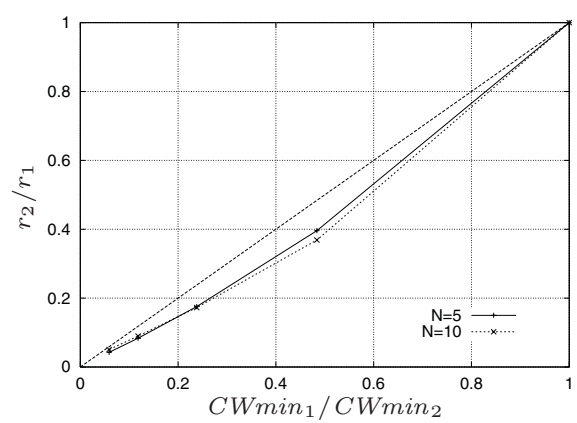

(a) CBR-over-UDP

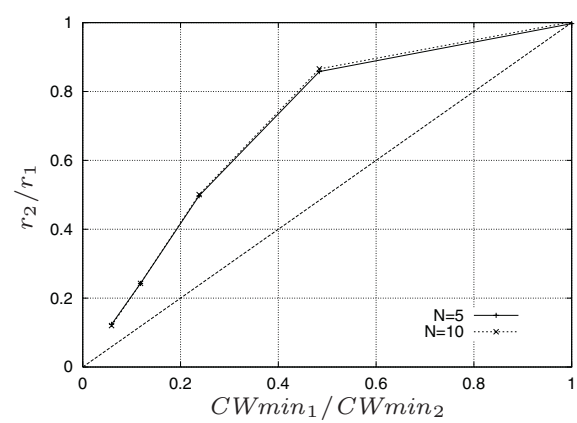

(b) TCP

Fig. 4. Throughput ratio $r_{2} / r_{1}$ as a function $C W m i n$ ratio $C W m_{i n} / C W m_{1} n_{2}$, for UDP and TCP traffic, and $N=5$ and $N=10$ sources.

inversely proportional to the $C W \min$ ratio. Fig. 4 (b) shows that the differentiation in the case of TCP traffic is less effective; see also [3]. Indeed, increasing the value of $C W$ min from 16 to 32 results in a throughput decrease of only $15 \%$. Also observe in Figs. 4)(a) and 4(b) that the effect of the number of connections on the throughput ratio as a function of the $C W m i n$ ratio is minimal, unlike DIFS differentiation with UDP traffic, Fig. 2(a).

Fig. 5(a) shows the average delay of voice traffic as a function of $C W m i n$, for $16,32,64,128$, and 256 time slots, in the case of 2 UDP connections carrying voice traffic, and $3 \mathrm{CBR}$ sources with an aggregate bandwidth of $30 \mathrm{Mbps}$. As

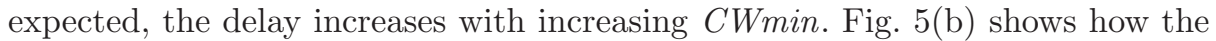
average delay of TCP traffic depends on $C W m i n$, in the case of 2 TCP connections, and $3 \mathrm{CBR}$ sources with an aggregate bandwidth of $30 \mathrm{Mbps}$. First observe that the delay in the TCP case is an order of magnitude smaller than in the UDP case. Furthermore, observe that for values of $C W$ min up to approximately 64 time slots, there is no increase of the average delay; indeed in this range the delay decreases with increasing $C W$ min. For values of $C W$ min above 64 time slots, the average delay increases with increasing $C W m i n$. 


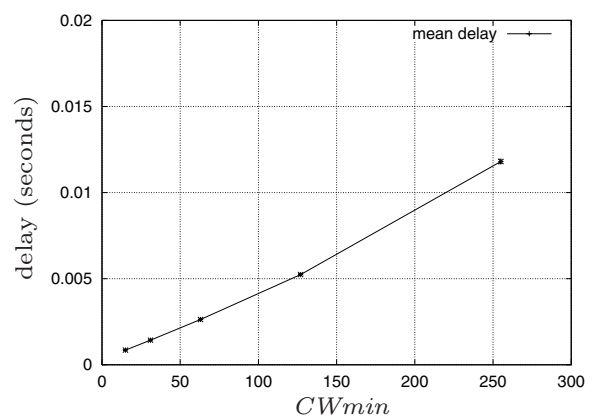

(a) Voice-over-UDP

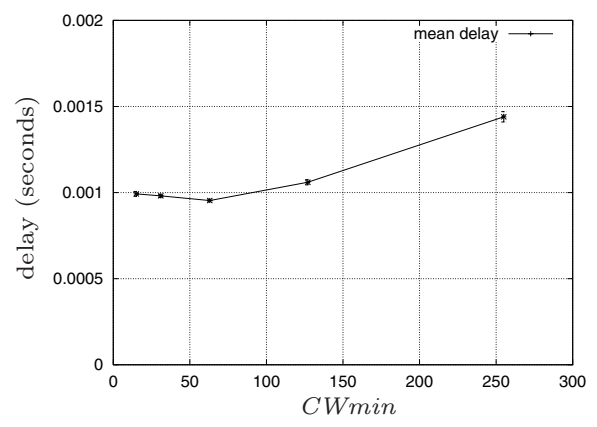

(b) TCP

Fig. 5. Delay differentiation for different $C W \min$ values.

\section{Algorithm for Assignment and Adjustment of $\mathrm{CWmin}$}

In the previous section we investigated the service differentiation that can be achieved with different values of $C W$ min. The optimal, in terms of efficient network utilization, values of $C W$ min will depend on the number of contending stations. In this section we describe an approach for assigning $C W m i n$ values to sources belonging to different classes, and for dynamically adjusting these values to achieve high aggregate throughput.

\subsection{Assignment of $C W \min$ Values}

Assume that different classes are associated with different weights, where a larger weight is assigned to a higher priority class. Based on the results of Section 2.3. the values of $C W$ min can be assigned inversely proportion to the weight of each class. Hence, if $\phi_{i}$ is the weight for class $i$, the value of the minimum contention window $C W \min _{i}$ for class $i$ is calculated using

$$
C W \min _{i}=\left\lfloor S F \cdot \frac{L i}{\phi_{i}}\right\rfloor,
$$

where $S F$ is some scaling factor and $L_{i}$ is the frame size; the frame size is added so that differentiation depends solely on the weight. In the case of collisions, the usual exponential backoff algorithm of 802.11 is performed.

The above assignment of CWmin values for different classes, based on the class' weight and the frame size, is similar to the calculation of the backoff interval in the scheme proposed in [5]; our approach differs in that we assign different values of $C W$ min rather than the backoff interval, and the behaviour of the exponential backoff algorithm in 802.11 remains the same. This presents

\footnotetext{
${ }^{1}$ More precisely, the left-hand side should be $C W \min _{i}-1$, since the backoff is selected from $[0, C W-1]$. slot_time. However, because we consider $C W \min \geq 16$, the difference in practice is insignificant.
} 


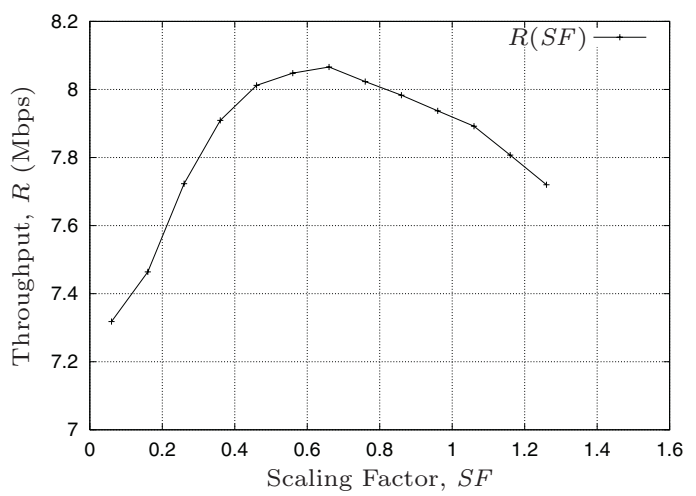

Fig. 6. Aggregate throughput as a function of the scaling factor. The measurement interval is $T_{m}=10$ seconds and the scaling factor step is $\Delta_{S F}=0.1$.

an important advantage, since our approach can be implemented solely at the access point of a WLAN, without requiring any changes at the wireless stations, assuming these support the IEEE 802.11e standard.

In the assignment of $C W m i n$ values, the value of the scaling factor is important, since it affects the channel utilization. Next we describe and evaluate an approach for adjusting the scaling factor, and subsequently the values of $C W m i n$, based on monitoring the actual throughput of the WLAN.

\subsection{Adaptive Recalculation of $C W m i n$}

The dependence of the aggregate throughput on the scaling factor is shown in Fig. 6. an identical result is presented in [5]. Observe that the throughput initially increases with the scaling factor up to a maximum value, after which it starts to decrease. Such a behaviour can be explained as follows: When the scaling factor is smaller than the optimum, the values of $C W \min$ are small, resulting in many collisions, hence the utilization and throughput is low. On the other hand, when the scaling factor gets too large, the values of $C W m i n$ are large, resulting in a large percentage of idle times, hence the wireless channel is underutilized.

Another important observation is that the optimum scaling factor is different for different network loads; this motivates the need to recalculate the scaling factor, hence the values of $C W m i n$, adaptively when the network load changes. From the shape of Fig. [6 this can be achieved by measuring the average throughput, and moving the scaling factor in the direction that increases the aggregate throughput. The details of the proposed procedure is shown in Fig. 7 . The procedure includes three parameters: the interval $T_{m}$ over which the throughput is measured, the scaling factor step size $\Delta_{S F}$, and the percentage $a$ used for deciding when to search for a new optimal scaling factor. A detailed discussion of how these factors affect the performance of the algorithm is contained in [10].

The above procedure for adjusting the scaling factor can be implemented at the access point. Moreover, each time the scaling factor changes, the access 
Step 1. The scaling factor is set to some initial value $S F_{1}$, and the aggregate throughput $R_{1}$ is measured at the access point (AP) over an interval $T_{m}$

Step 2. $S F_{2}:=S F_{1}+\Delta_{S F}$, and the aggregate throughput $R_{2}$ is measured at the AP

Step 3. If $R_{2}>R_{1}$ then $S F$ increases with step $\Delta_{S F}$ while the aggregate throughput increases else if $R_{2}<R_{1}$ then $S F$ decreases with step $\Delta_{S F}$ while the aggregate throughput increases

Step 4. Let $S F^{*}$ be the optimal scaling factor, and $R^{*}$ the throughput when Step 3 ends Let $S F_{1}:=S F^{*}$

For the same scaling factor, the AP continuously measures the average throughput $R_{1}$ If $R_{1}<a R^{*}$, where $a \in(0,1)$ then goto Step 2

Fig. 7. Procedure for adjusting the scaling factor $S F$.

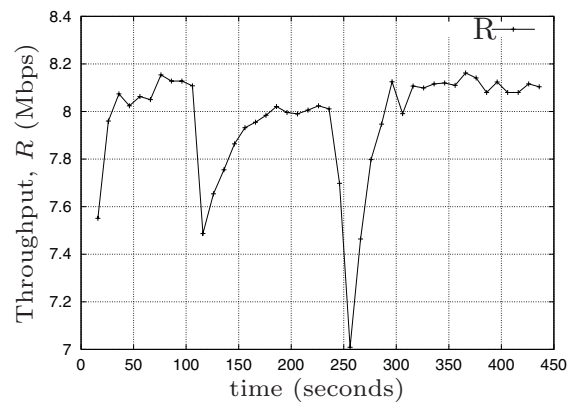

(a) Exponential

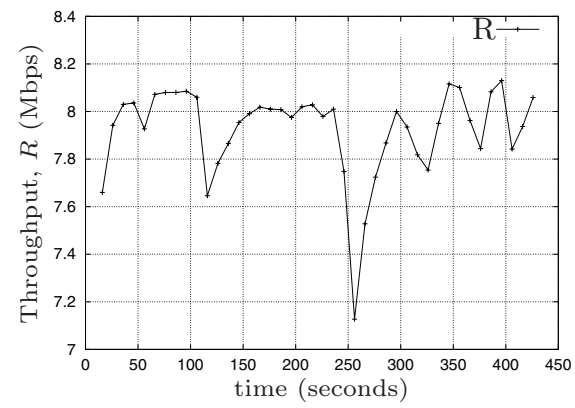

(b) Pareto

Fig. 8. Results for exponential and pareto traffic sources. Exponential: average "on" 800 ms, "off" $200 \mathrm{~ms}$. Pareto: same average on/off, and shape parameter 1.5.

point would recalculate, using Eq. 1, the value of $C W$ min $_{i}$ for each class $i$, and distribute it to the wireless stations; this communication can utilize the particular procedure that will be used in IEEE 802.11e.

\subsection{Simulation Results}

In this section we demonstrate the operation of the procedure described in the previous section for dynamically adjusting $C W m i n$. The values for the three parameters of the procedure we consider are the following, see 10, for a justification of this selection: throughput measurement interval $T_{m}=10$ seconds, scaling factor step size $\Delta_{S F}=0.1$, and throughput decrease threshold $a=0.95$.

Initially, the WLAN has 13 stations, each producing exponential (Fig. 8(a)) or pareto $(\mathrm{Fig} 8(\mathrm{~b}))$ traffic with rate $1 \mathrm{Mbps}$. At time 130 seconds, 20 more identical stations enter the WLAN; at time 240 seconds, 23 of the stations depart, leaving 10 stations in the WLAN. Observe that the procedure for adjusting $C W$ min kicks in whenever the number of stations changes, and tries to maintain a high throughput. The maximum throughput is approximately $8 \mathrm{Mbps}$, with the exact value depending on the number of stations. Indeed, a smaller number of stations can achieve a larger aggregate throughput; this is due to the smaller probability of collisions when there are fewer stations. The results from these fig- 
ures show that, for the particular parameter values, the procedure's convergence time is of the order of 10 s of seconds, which is sufficient in environments where the number of wireless users change over timescales larger than a few minutes.

\section{Conclusion}

In this paper we first presented simulation experiments on service differentiation, in terms of both throughput and delay, using various 802.11 MAC layer parameters, namely the maximum frame size, the minimum contention window, and the DCF inter-frame spacing interval. Second, we proposed a simple yet effective procedure for adjusting the minimum contention window based on actual throughput measurements. An important advantage of our approach is that it can be implemented solely at the access point; the wireless stations are only required to support the emerging IEEE 802.11e standard. Our initial experiments show that the approach is quite robust, and can effectively adjust the contention window to achieve high aggregate throughput. Further investigations focus on quantifying the tradeoffs of the procedure's parameters; such information will be useful for tuning these parameters in a real environment. Furthermore, the procedure's reactivity should depend on the timescales over which the network conditions change significantly; identifying these timescales can assist in tuning the procedure's degree of reactivity.

\section{References}

1. IEEE: Draft Supplement - Part 11: MAC Enhancements for QoS, IEEE 802.11e/ D2.0 (2001)

2. Aad, I., Castelluccia, C.: Differentiation mechanisms for IEEE 802.11. In: Proc. of IEEE INFOCOM'01 (2001)

3. Aad, I., Castelluccia, C.: Remarks on per-flow differentiation in IEEE 802.11. In: Proc. of European Wireless 2002

4. Bharghavan, V., Demers, A., Shenker, S., Zhang, L.: MACAW: a media access protocol for wireless LANs. In: Proc. of ACM SIGCOMM'94 (1994)

5. Vaidya, N.H., Bahl, P., Gupta, S.: Distributed fair scheduling in a wireless LAN. In: Proc. of ACM MOBICOM'00 (2000)

6. Bensaou, B., Wang, Y., Ko, C.C.: Fair medium access in 802.11 based wireless ad-hoc networks. In: Proc. of ACM MOBIHOC'02 (2002)

7. Qiao, D., Shin, K.G.: Achieving efficient channel utilization and weighted fairness for data communications in IEEE 802.11 WLAN under DCF. In: Proc. of IEEE/IFIP IWQoS'02 (2002)

8. Veres, A., Campbell, A.T., Barry, M., Sun, L.H.: Supporting service differentiation in wireless packet networks using distributed control. IEEE J. Select. Areas in Commun. 19 (2001) 2094-2104

9. UCB/LBNL/VINT: Network Simulator - ns (version 2). (http://www-mash.cs.berkeley.edu/ns.html)

10. Siris, V.A., Kavouridou, M.: Achieving service differentiation and high utilization in IEEE 802.11. Technical Report No. 322, ICS-FORTH (2003) 